\title{
Simulated Galactic methanol maser distribution to constrain Milky Way parameters
}

\author{
L. H. Quiroga-Nuñez ${ }^{1,2}$, H. J. van Langevelde ${ }^{2,1}$, M. J. Reid ${ }^{3}$, and J. A. Green ${ }^{4,5}$ \\ ${ }^{1}$ Leiden Observatory, Leiden University, PO Box 9513, 2300 RA Leiden, The Netherlands \\ e-mail: quiroganunez@strw.leidenuniv.nl \\ 2 Joint Institute for VLBI ERIC (JIVE), Postbus 2, 7990 AA Dwingeloo, The Netherlands \\ 3 Harvard-Smithsonian Center for Astrophysics, 60 Garden Street, Cambridge, MA 02138, USA \\ ${ }_{5}^{4}$ CSIRO Astronomy and Space Science, Australia Telescope National Facility, PO Box 76, Epping, NSW 1710, Australia \\ 5 SKA Organisation, Jodrell Bank Observatory, Lower Withington, Macclesfield, SK11 9DL, UK
}

Received 22 February 2017 / Accepted 7 June 2017

\begin{abstract}
Context. Using trigonometric parallaxes and proper motions of masers associated with massive young stars, the Bar and Spiral Structure Legacy (BeSSeL) survey has reported the most accurate values of the Galactic parameters so far. The determination of these parameters with high accuracy has a widespread impact on Galactic and extragalactic measurements.

Aims. This research is aimed at establishing the confidence with which such parameters can be determined. This is relevant for the data published in the context of the BeSSeL survey collaboration, but also for future observations, in particular from the southern hemisphere. In addition, some astrophysical properties of the masers can be constrained, notably the luminosity function.

Methods. We have simulated the population of maser-bearing young stars associated with Galactic spiral structure, generating several samples and comparing them with the observed samples used in the BeSSeL survey. Consequently, we checked the determination of Galactic parameters for observational biases introduced by the sample selection.

Results. Galactic parameters obtained by the BeSSeL survey do not seem to be biased by the sample selection used. In fact, the published error estimates appear to be conservative for most of the parameters. We show that future BeSSeL data and future observations with southern arrays will improve the Galactic parameters estimates and smoothly reduce their mutual correlation. Moreover, by modeling future parallax data with larger distance values and, thus, greater relative uncertainties for a larger numbers of sources, we found that parallax-distance biasing is an important issue. Hence, using fractional parallax uncertainty in the weighting of the motion data is imperative. Finally, the luminosity function for $6.7 \mathrm{GHz}$ methanol masers was determined, allowing us to estimate the number of Galactic methanol masers.
\end{abstract}

Key words. masers - astrometry - Galaxy: fundamental parameters - Galaxy: kinematics and dynamics - Galaxy: structure

\section{Introduction}

A lack of accurate distance measurements throughout the Galaxy combined with our location within the Milky Way have complicated the interpretation of astrometric measurements (Reid \& Honma 2014). Consequently, the most fundamental Galactic parameters, such as the distance to the Galactic center $\left(R_{0}\right)$, the rotation speed at the solar radius $\left(\Theta_{0}\right)$, and the rotation curve (e.g., $\mathrm{d} \Theta / \mathrm{d} R$ ) have not been established with high accuracy. At Galactic scales, distance estimates through radial velocities, mass and luminosity calculations of sources within the Galaxy, as well as the mass and luminosity estimates of the Milky Way, depend on the Galactic parameters. Additionally, extragalactic measurements are based on Galactic calibrations that are made using the Milky Way parameter values. Therefore, highly accurate estimates of the fundamental Galactic parameters are vitally important.

A step forward came with the HIPPARcos satellite (Perryman et al. 1997). It provided astrometric accuracies of the order of 1 milliarcs (mas), which allows distance estimations in the solar neighborhood $(\sim 100 \mathrm{pc})$ with $10 \%$ accuracy. However, this is a tiny portion of the Milky Way. The ongoing European Space Agency mission, Gaia, aims to measure parallaxes and proper motions of $10^{9}$ stars with accuracies up to $20 \mu$ as at 15 mag with a distance horizon of $5 \mathrm{kpc}$ with $10 \%$ accuracy and $10 \mathrm{kpc}$ with $20 \%$ accuracy (Perryman et al. 2001; Gaia Collaboration 2016). Although Gaia will transform our knowledge of the Milky Way, the mission is restricted to optical wavelengths and due to significant dust obscuration, it will not be able to probe the Galactic plane freely. In contrast, radio wavelengths are not affected by dust extinction and can be used throughout the Galaxy.

Direct accurate distances and proper motions have been measured for maser-bearing young stars (e.g. Sanna et al. 2014; Burns et al. 2017); this data was obtained employing Very Long Baseline Interferometry (VLBI). This astrometric information has provided us with a better understanding of the Milky Way's spiral structure, insights into the formation and evolution of our Galaxy, its 3D gravitational potential, and the Galactic baryonic and dark matter distribution (Efremov 2011).

The most suitable radio beacons for astrometry are methanol (6.7 and $12.2 \mathrm{GHz})$ and water $(22 \mathrm{GHz})$ masers (Brunthaler et al. 2011). In addition to being bright, water masers can be associated with high mass star forming regions (HMSFRs), while class II 6.7 and $12 \mathrm{GHz}$ methanol masers are uniquely associated with HMSFRs (e.g. Breen et al. 2013; Surcis et al. 2013). By detecting $6.7 \mathrm{GHz}$ methanol masers, we trace the Galactic 
spiral structure because HMSFRs are expected to be born close to a spiral arm and evolve more quickly than low-mass stars (Yusof et al. 2013). Therefore, HMSFRs should follow the disk rotation with low dispersion (compared, for example, to masers in evolved stars).

Given parallax, proper motion measurements, source coordinates, and line-of-sight velocities (from Doppler shifts of spectral lines) to methanol and water masers, it is possible to sample complete phase-space information. This provides direct and powerful constraints on the fundamental parameters of the Galaxy. The Bar and Spiral Structure Legacy (BeSSeL ${ }^{1}$ ) survey has addressed this task using different arrays: the Very Long Baseline Array (VLBA) in USA and the European VLBI Network (EVN) in Europe, Asia and South Africa. Additionally similar parallax and proper motion data has come from the VLBI Exploration of Radio Astrometry (VERA) in Japan. The most recent summary paper (Reid et al. 2014) lists astrometric data for 103 parallax measurements with typical accuracies of $20 \mu$ as. By fitting these sources to an axially symmetric Galactic model, they provide accurate values for the fundamental Galactic parameters: $R_{0}=8.34 \pm 0.16 \mathrm{kpc}, \Theta_{0}=240 \pm 8 \mathrm{~km} \mathrm{~s}^{-1}$, and $\mathrm{d} \Theta / \mathrm{d} R=-0.2 \pm 0.4 \mathrm{~km} \mathrm{~s}^{-1} \mathrm{kpc}^{-1}$ between Galactocentric radii of 5 and $16 \mathrm{kpc}$.

Although the BeSSeL survey data is very accurate, the target selection used was necessarily biased. It has targeted the brightest known masers accessible to the (northern hemisphere) VLBI arrays used. Most of the published targets used by BeSSeL for astrometric measurements are $22 \mathrm{GHz}$ water masers and $12 \mathrm{GHz}$ methanol masers that were originally selected based on $6.7 \mathrm{GHz}$ surveys. In the current study, a model used to simulate the $6.7 \mathrm{GHz}$ methanol maser distribution in the Milky Way is presented. The model was compared with systematic surveys, allowing us to determine the luminosity function. Also, it is used to generate different artificial samples that can be used to test how accurately they can fit a Galactic model and how a given level of incompleteness can bias the Galactic parameter values. This is particularly important when more sources are being added to the BeSSeL sample.

In Sect. 2 the components and assumptions of the model are presented. Next, Sect. 3 describes the luminosity function fitted using observational surveys, the Galactic parameter results, and the correlation among parameters using several samples. Finally, the discussion and conclusions of the results compared to the BeSSeL findings are shown in Sects. 4 and 5, respectively.

\section{Model for the $6.7 \mathrm{GHz}$ methanol maser distribution in the spiral structure}

The main components of the Milky Way can be identified as a halo, nuclear bulge (or bar), and two disk components: a thin and a thick disk (see e.g. Gilmore \& Reid 1983; Rix \& Bovy 2013). The current model is centered on the thin disk component, more specifically on a spiral structure between $3 \mathrm{kpc}$ and $15 \mathrm{kpc}$ as traced by HMSFRs that contains methanol maser bearing stars. Following the analysis made by Reid et al. (2014), the model is based on a galaxy with spiral structure. The analysis of the rotation and scale of the galaxy does not seem directly dependent on this assumption.

The aim of the model is to build a simulated database ready to be processed with the Galactic parameter fitting method used by the BeSSeL survey. To do this, each simulated $6.7 \mathrm{GHz}$ methanol maser has spatial coordinates, velocity components,

\footnotetext{
1 http://bessel.vlbi-astrometry.org/
}

Table 1. Spatial, velocity, and luminosity distributions used in the current model.

\begin{tabular}{ccc}
\hline \hline $\begin{array}{c}\text { Model } \\
\text { variable }\end{array}$ & $\begin{array}{c}\text { Distribution } \\
\text { type }\end{array}$ & $\begin{array}{c}\text { Distribution } \\
\text { parameters }\end{array}$ \\
\hline Galactic & Radial decay and & $h_{\mathrm{r}}=2.44 \mathrm{kpc}(1)$ \\
plane $(X, Y)$ & Monte Carlo rejection & $\sigma_{d}=0.35 \mathrm{kpc}(1)$ \\
\hline Vertical & Gaussian & $\mu_{z}=0 \mathrm{kpc}$ \\
position $(Z)(2)$ & & $\sigma_{z}=25 \mathrm{pc}$ \\
\hline Radial & Gaussian & $\mu_{\mathrm{r}}=0 \mathrm{~km} \mathrm{~s}^{-1}$ \\
velocity $(U)$ & & $\sigma_{\mathrm{r}}=5 \mathrm{~km} \mathrm{~s}^{-1}$ \\
\hline Tangential & Gaussian & $\mu_{\mathrm{t}}=\Theta_{0}=240 \mathrm{~km} \mathrm{~s}^{-1}(1)$ \\
velocity $(V)$ & & $\sigma_{\mathrm{t}}=9 \mathrm{~km} \mathrm{~s}^{-1}$ \\
\hline Vertical & Gaussian & $\mu_{\mathrm{v}}=0 \mathrm{~km} \mathrm{~s}^{-1}$ \\
velocity $(W)$ & & $\sigma_{\mathrm{v}}=5 \mathrm{~km} \mathrm{~s}^{-1}$ \\
\hline Luminosity & Power & Cutoffs: $10^{-8} L_{\odot}, 10^{-3} L_{\odot}$ \\
function $(L)(3)$ & law & and $\alpha=-1.43$
\end{tabular}

Notes. We assumed the sun's vertical position to be $z=0 \mathrm{pc}$ and any change in this value, which Goodman et al. (2014) suggested to be $25 \mathrm{pc}$, was found negligible at these scales.

References. (1) Reid et al. (2014); (2) Green \& McClure-Griffiths (2011); and (3) Pestalozzi et al. (2007). Radial, tangential and vertical velocity dispersion values are discussed in Sect. 2.3.

and an associated intrinsic luminosity (and their respective uncertainties). In the following subsections, we explain each of the distributions and the initial parameters adopted, as well as the fitting procedure used to obtain the Galactic parameters from the astrometric data. Table 1 presents a summary of the distributions and values used.

\subsection{Initial parameters}

Reid et al. (2014) presented their best estimates of the Galactic parameter values (Model A5), which we adopt here (see Table 2):

- $R_{0}, \Theta_{0}, \mathrm{~d} \Theta / \mathrm{d} R$ : fundamental Galactic parameters. We took the current results of the BeSSeL survey, which assumes a Galactic model as a disk rotating at a speed of $\Theta(R)=\Theta_{0}+$ $\frac{\mathrm{d} \Theta}{\mathrm{d} R}\left(R-R_{0}\right)$.

- $\bar{U}_{\mathrm{s}}, \bar{V}_{\mathrm{s}}$ : average source peculiar motion. When velocities are measured, systematic extra velocity components can appear as a result of two effects: gas approaching a spiral arm with enhanced gravitational attraction and magnetohydrodynamic shocks as the gas enters the arm; therefore, these extra velocity components, which are defined at the position of each source, account for any average peculiar motion of the masers.

- $U_{\odot}, V_{\odot}, W_{\odot}$ : solar motion. Because the model predicts the velocities with respect to the local standard of rest (LSR) for all masers, the solar motion must be taken into account in order to make the proper heliocentric corrections.

- N: number of sources. The total number of $6.7 \mathrm{GHz}$ methanol masers in the Galaxy is a required parameter to populate the spiral arms. In Sect. 3, this parameter is fitted by comparing the model with the results of Methanol Multibeam Survey (MMB, see: Green et al. 2009, 2010, 2012; Caswell et al. 2010, 2011) results given the adopted spatial distribution (Sect. 2.2) and luminosity function (Sect. 2.5). 

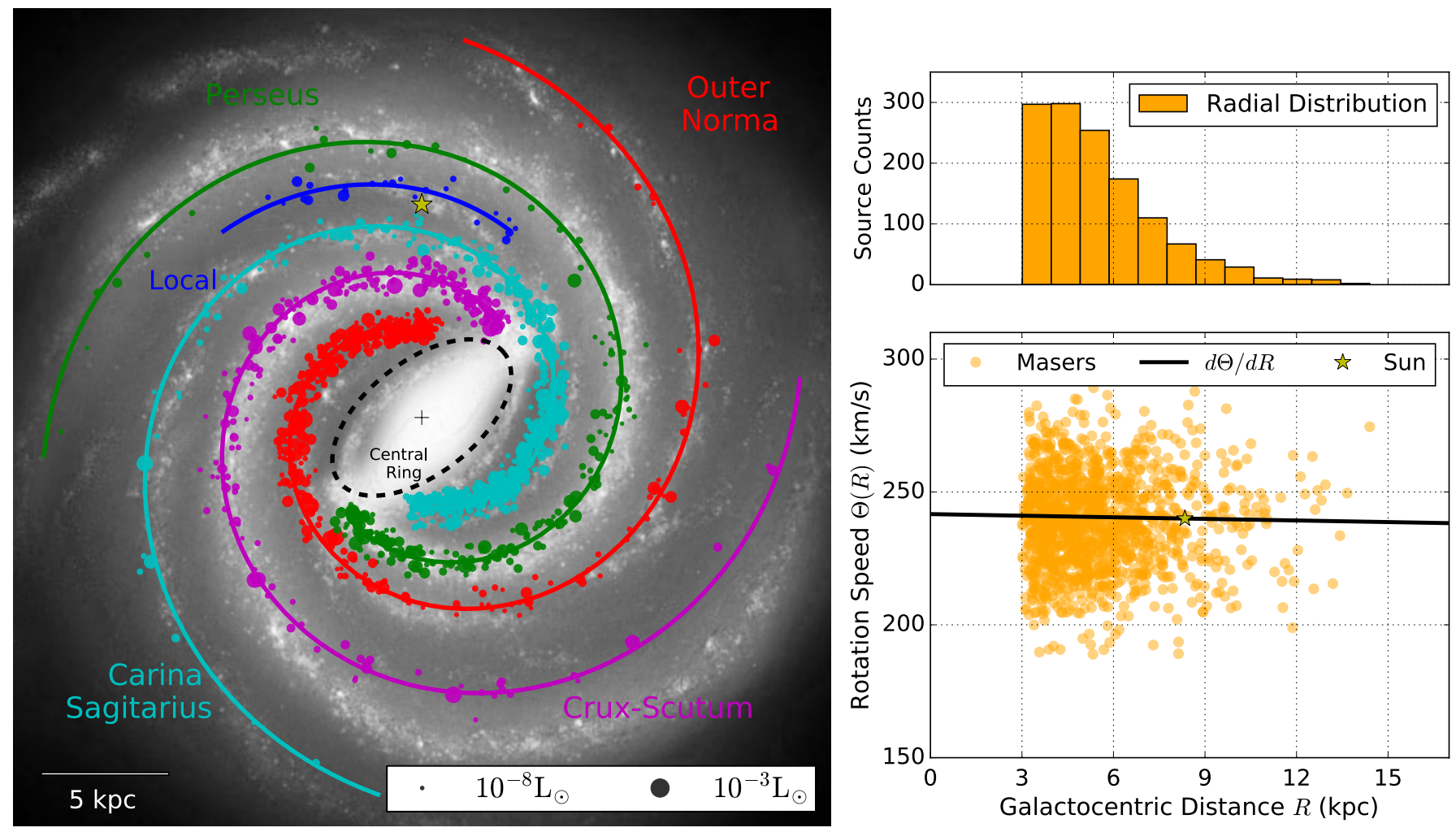

Fig. 1. Left: galactic plane distribution of $6.7 \mathrm{GHz}$ methanol masers seen from the NGP overlaid on an artist impression of the Milky Way (R. Hurt: NASA/JPLCaltech/SSC). The spiral structure was constructed following Wainscoat et al. (1992) and the central molecular ring or $3 \mathrm{kpc}$ arms (Green et al. 2011) was indicated, but it is not part of the model. The simulated spatial maser distribution is presented in Sect. 2.2. The plot also includes the intrinsic peak luminosity for each source as the point size following the luminosity function described in Sect. 2.5. In this figure, the Galaxy rotates clockwise. Bottom right: tangential velocity distribution as a function of Galactocentric distance for the simulated $6.7 \mathrm{GHz}$ methanol masers. It also displays the rotation curve, $\mathrm{d} \Theta / \mathrm{d} R=-0.2 \mathrm{~km} \mathrm{~s}^{-1} \mathrm{kpc}^{-1}$. Top right: radial distribution of the $6.7 \mathrm{GHz}$ methanol masers for our model is also shown. Table 1 presents a summary of the distributions used.

Table 2. Description of initial parameters values used in the model which are based on the Model A5 results published in Reid et al. (2014).

\begin{tabular}{ccc}
\hline \hline Parameter & Definition & Value \\
\hline$R_{0}$ & Sun-Galactocentric distance & $8.34 \mathrm{kpc}$ \\
$\Theta_{0}$ & Solar rotation speed & $240 \mathrm{~km} \mathrm{~s}^{-1}$ \\
$\mathrm{~d} \Theta / \mathrm{d} R$ & Rotation curve & $-0.2 \mathrm{~km} \mathrm{~s}^{-1} \mathrm{kpc}^{-1}$ \\
$U_{\odot}$ & Inward radial solar velocity & $10.7 \mathrm{~km} \mathrm{~s}^{-1}$ \\
$V_{\odot}$ & Tangential solar velocity & $15.6 \mathrm{~km} \mathrm{~s}^{-1}$ \\
$W_{\odot}$ & Vertical solar velocity & $8.9 \mathrm{~km} \mathrm{~s}^{-1}$ \\
$\bar{U}_{\mathrm{s}}$ & Inward radial average peculiar motion & $2.9 \mathrm{~km} \mathrm{~s}^{-1}$ \\
$\bar{V}_{\mathrm{s}}$ & Tangential average peculiar motion & $-1.5 \mathrm{~km} \mathrm{~s}^{-1}$ \\
\hline
\end{tabular}

\subsection{Spatial distribution}

The spatial distribution along the spiral arms can be split into two components, a Galactic plane distribution and a vertical component distribution $(Z)$. The latter can be drawn using a random generator from a Gaussian distribution with a mean of $0 \mathrm{pc}$ and $\sigma=25 \mathrm{pc}$ since massive young stars are found to be born close to the Galactic plane (see e.g. Green \& McClure-Griffiths 2011; Bobylev \& Bajkova 2016).

The Galactic plane distribution is drawn following two constraints. First, the density of HMSFRs falls off exponentially with the Galactocentric distance $(R)$ (Bovy \& Rix 2013), and second, each source should be associated with a spiral arm (Reid et al. 2014). For the first constraint, the maser radial distribution follows Cheng et al. (2012)

$n(R) \propto \mathrm{e}^{-R / h_{R}}$,

where $n(R)$ is the number of sources and $h_{R}$ the exponential scale length, which has been estimated from the maser parallax data assuming a Persic Universal rotation curve formulation to be $2.44 \mathrm{kpc}$ (Reid et al. 2014) which we assumed valid for massive young stars. The top right panel of Fig. 1 shows the radial distribution of the simulated masers.

For the second constraint, the spiral arm positions were set following an analytic approximation made by Wainscoat et al. (1992). Each spiral arm (four main arms and the local arm) can be located in the Galactic plane using a simple relation in polar coordinates. The left plot of Fig. 1 depicts the position of the spiral arms as seen from the north galactic pole (NGP). In order to populate the spiral arms with $6.7 \mathrm{GHz}$ methanol masers, a rejection sampling Monte Carlo method was implemented. For this, the model takes a source from the radial distribution (Eq. (1)) and then the distance is calculated between the source and the closest spiral arm. That distance $d$ is evaluated in a probability density function of a Gaussian distribution

$P(d) \propto \exp \left(\frac{-(d-\mu)^{2}}{2 \sigma_{\mathrm{d}}^{2}}\right)$,

where $\mu=0 \mathrm{kpc}$, yielding the same likelihood of the source to be behind or in front of the spiral arm. We took $\sigma_{d}=0.35 \mathrm{kpc}$, which corresponds to the maximum spiral width arm observed 


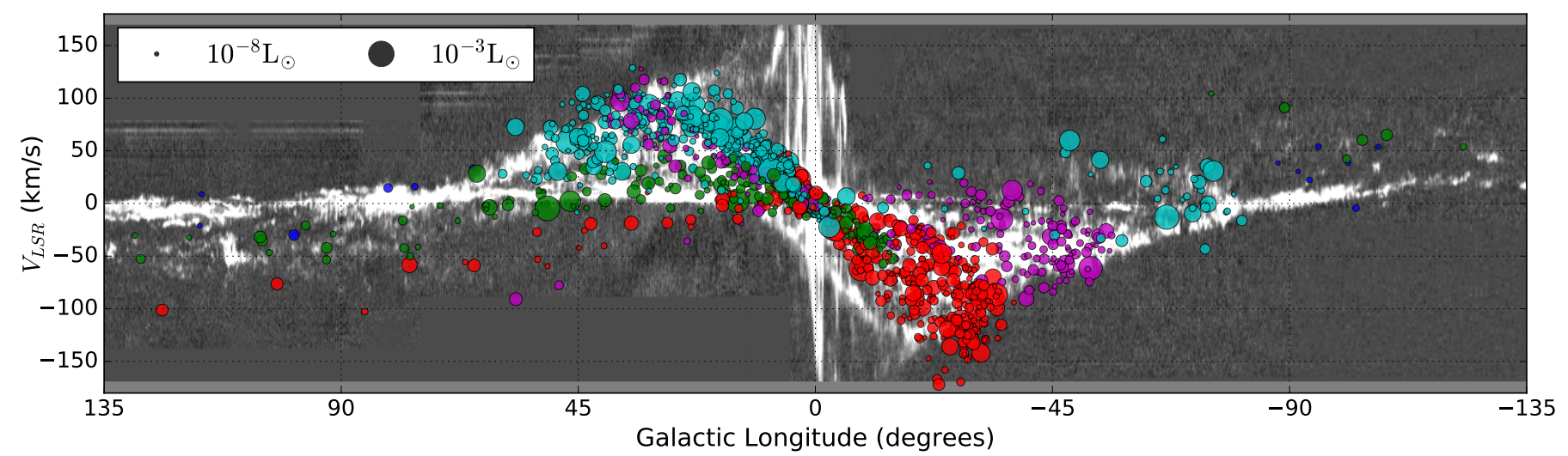

Fig. 2. Velocity with respect to the LSR as a function of the Galactic longitude for the simulated $6.7 \mathrm{GHz}$ methanol masers distribution. The point size is a measure of the peak luminosity function (Sect. 2.5). Masers associated with different spiral arms are color-coded as in Fig. 1. The figure is overlaid on the CO emission $(J=1-0)$ plotted in grayscale and taken from Dame et al. (2001).

for HMSFRs (Reid et al. 2014). The model evaluates $P(d)$ for each source and compares it with a random value $k(0<k<1)$. If $k>P(d)$, the source is rejected and the model takes another source from the radial distribution to calculate $P(d)$ again and compare it with a new $k$. However, if a source satisfies $k<P(d)$, then the source is taken as a part of the model. The acceptance process will continue until it reaches the total number of sources $(N)$. One example of a resulting spatial distribution can be seen in Figs. 1 and 2.

\subsection{Velocity distribution}

For the velocity distribution, we used a cylindrical coordinate system $(U, V, W)$ in a rotational frame with an angular velocity of $\Theta(R)$ in the direction of the Galaxy rotation, i.e., clockwise seen from the NGP. In this system, $U$ is the radial component defined positive towards the center of the Galaxy, $V$ is the tangential velocity component defined positive in the direction of the Galactic rotation and $W$ is the vertical velocity component defined positive towards the NGP.

We drew Gaussian distributions for each velocity component independently using the values, distributions and dispersions related in Table 1. For the tangential velocity, we adopted a Gaussian distribution with a mean value given by $\Theta(R)=$ $\Theta_{0}+\mathrm{d} \Theta / \mathrm{d} R\left(R-R_{0}\right)$ and a dispersion of $\sigma_{\mathrm{t}}=9 \mathrm{~km} \mathrm{~s}^{-1}$ (see Table 1). The values for $\Theta_{0}, \mathrm{~d} \Theta / \mathrm{d} R$ and $R_{0}$ are provided in Table 2. The lower right panel of Figs. 1 and Fig. 2 show the distribution of the Galactic tangential velocities $\Theta(R)$ and the maser velocities with respect to LSR as a function of Galactocentric distance and Galactic longitude respectively, assuming the values listed in Table 2. The adopted dispersions for radial and vertical velocity components $\left(\sigma_{\mathrm{r}, \mathrm{v}}=5 \mathrm{~km} \mathrm{~s}^{-1}\right)$ are consistent with our estimates of virial motions of individual massive stars, based on BeSSeL data, whereas $\sigma_{\mathrm{t}}$ was set larger to allow for the possible effects of gravitational accelerations in the presence of material near spiral arms.

\subsection{Methanol masers represented in the model}

The BeSSeL survey determined proper motions and parallaxes of water masers (at $22 \mathrm{GHz}$ ) and methanol masers (at 6.7 and $12 \mathrm{GHz}$ ) and fit them to an axially symmetric Galactic model to estimate the Galactic parameters. Compared with the BeSSeL survey, we have made a simplification by assuming that all sources are selected from $6.7 \mathrm{GHz}$ methanol masers surveys, but observed with VLBI at $12 \mathrm{GHz}$.

\subsection{Luminosity distribution}

Notably, for our model it is important to estimate astrometric observational errors based on maser detectability, which are directly related to the peak flux density $\left(S_{\mathrm{p}}\right)$ of each maser, i.e., the flux density emitted in a specific line integrated over a single channel width. The peak flux density function can be estimated if the peak luminosity function and the spatial distribution are known, assuming isotropic emission. Although the individual maser spots may not radiate isotropically, we assume that this holds over the sample of randomly oriented masers.

Pestalozzi et al. (2007) have suggested that the $6.7 \mathrm{GHz}$ methanol maser luminosity distribution takes the form of a single power law with sharp cutoffs of $10^{-8} L_{\odot}$ and $10^{-3} L_{\odot}$ and a slope $(\alpha)$ between -1.5 and -2 . We assume the same dependence for the peak luminosity function (see Fig. 3), but we refine it by varying the parameters to match the results of the MMB survey. The results of this procedure are presented in Sect. 3.1.

\subsection{Error allocation}

In order to be able to use simulated data in tests to estimate the Galactic parameters, it is necessary to assign observational error distributions. For our model, the errors in the parallax and proper motions were estimated following a calculation for relative motions of maser spots and statistical parallaxes, i.e., $\sigma_{\pi} \propto \Theta_{\text {res }} /(S / N)$ and $\sigma_{\mu_{\alpha, \delta}}=\sigma_{\pi} /(1 \mathrm{yr})$, where $\Theta_{\text {res }}$ is the VLBA resolution for $12 \mathrm{GHz}$ methanol masers. The signal-to-noise ratio $(\mathrm{S} / \mathrm{N})$ depends on the peak flux density value $\left(S_{\mathrm{p}}\right)$ and given that most of the current data of the BeSSeL survey are based on VLBA observations, we adopted a channel width of $50 \mathrm{kHz}$ $\left(1.24 \mathrm{~km} \mathrm{~s}^{-1}\right)$ at $12 \mathrm{GHz}$ and an integration time of $2 \mathrm{~h}$. This was used to estimate the $\mathrm{S} / \mathrm{N}$ and thus the errors in parallax and proper motions. Reid et al. (2014) estimated an additional error term for $\sigma_{V_{\text {los }}}\left(5 \mathrm{~km} \mathrm{~s}^{-1}\right)$, which is associated with the uncertainty on transferring the maser motions to the central star. This error dominates the BeSSeL observations of $V_{\text {los }}$, and this uncertainty is reflected in the value of $\sigma_{V_{\text {los }}}$.

Parallax estimates in Reid et al. (2014) are often dominated by residual, whereas troposphere-related errors dominate in the astrometry, and so we adopted a simple prescription for parallax 

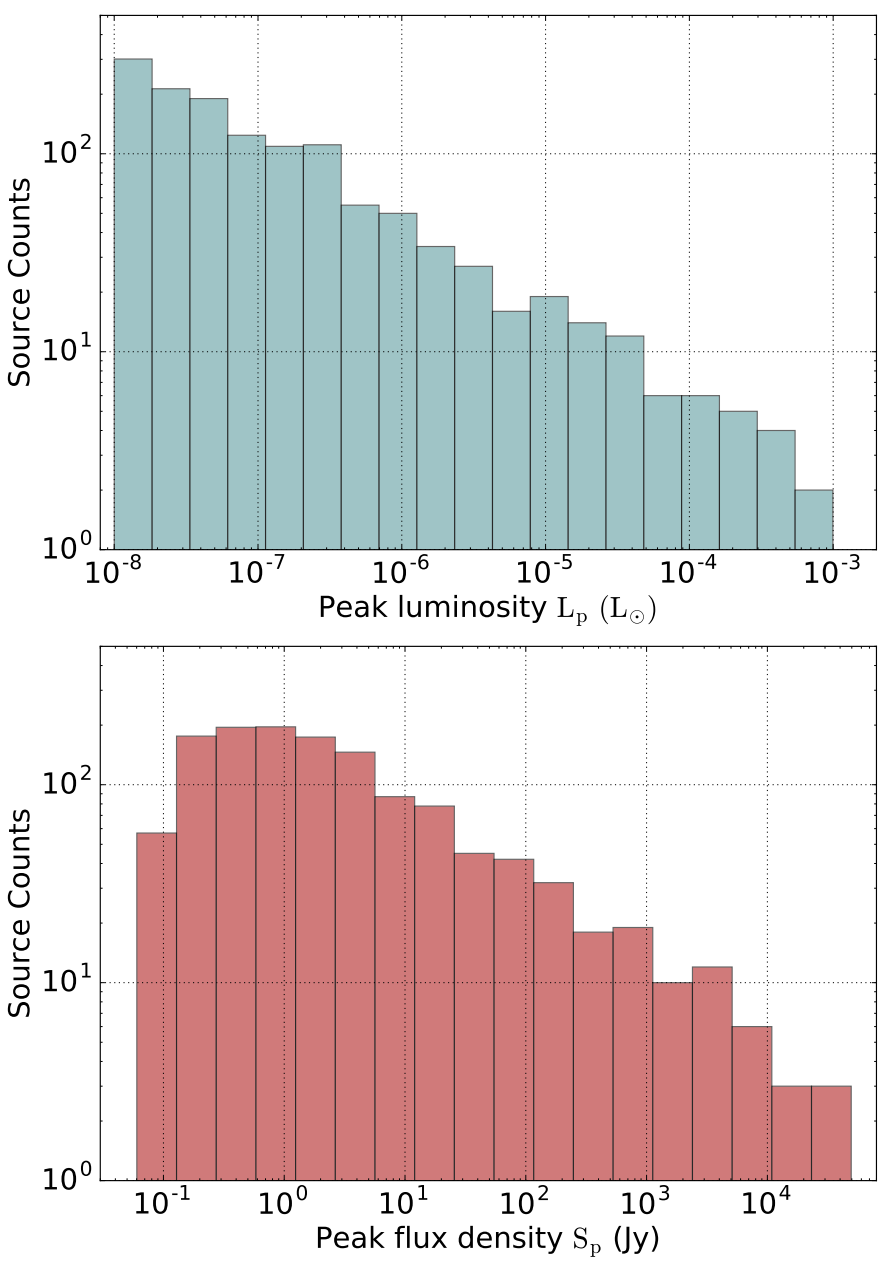

Fig. 3. Top: peak luminosity function adopted in the model using the fitted values for the total number of $6.7 \mathrm{GHz}$ methanol masers $(N=1300)$ and the slope of the luminosity function $(\alpha=-1.43)$, see Sects. 2.5 and 3.1. Bottom: peak flux density function obtained without sensitivity limit.

uncertainty (as shown above), which does not directly include systematic effects. However, when a large number of simulated sources are used, many weak masers are included that would be $\mathrm{S} / \mathrm{N}$ limited. Figure 4 shows a comparison between the two error distributions for observational and simulated parallax measurements in which our $\mathrm{S} / \mathrm{N}$ error estimate yields a similar distribution to the uncertainties used in Reid et al. (2014).

The fitting procedure described by Reid et al. (2014) used to determine the Galactic parameters (combining BeSSeL and VERA data) requires high accuracy VLBI data as input. This data consists of a 3D position vector $(\alpha, \delta, \pi)$, a 3D velocity vector $\left(\mu_{\alpha}, \mu_{\delta}, V_{\text {los }}\right)$, and the errors $\sigma_{\pi}, \sigma_{\mu_{\alpha}}, \sigma_{\mu_{\delta}}$ and $\sigma_{V_{\text {los }}}$. Although the model gives exact values for position and velocities of each maser source seen from the Earth, we are interested in realistic values as input for the fitting procedure. Therefore, we add a noise component to each observable quantity $\left(\pi, \mu_{\alpha}, \mu_{\delta}\right.$, $V_{\text {los }}$ ), using random values following Gaussian distributions with standard deviations equal to the estimated errors previously calculated. By changing the error distribution, we can control the quality of the data entered in the fitting procedure.

\subsection{Fitting procedure}

The fitting procedure used was adopted from the BeSSeL survey (see Reid et al. 2009, 2014). The input data for the fitting

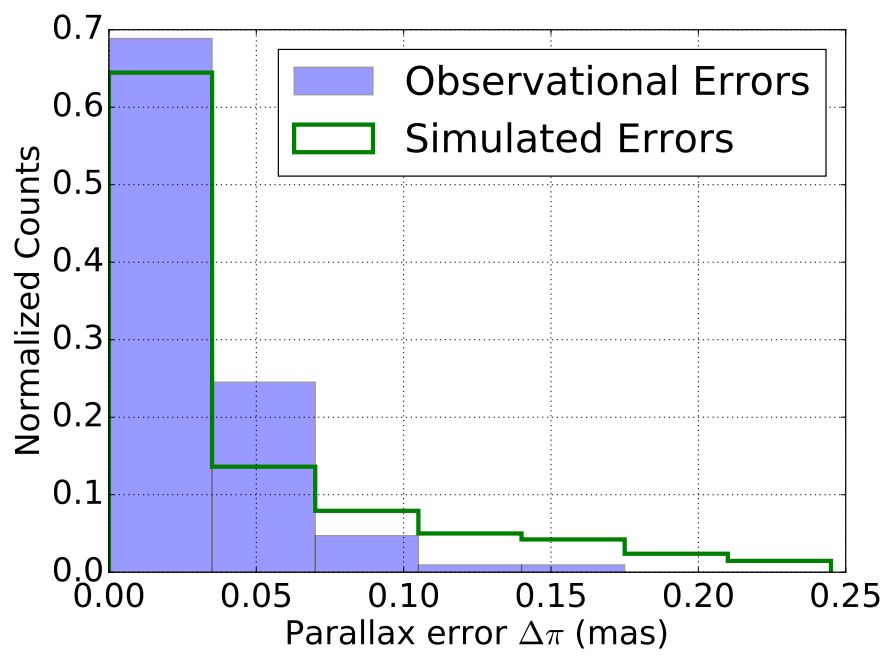

Fig. 4. Comparison between the error distribution for observational and simulated parallax measurements. Observational errors are based on 103 astrometric sources published in Reid et al. (2014) as part of the BeSSeL survey.

procedure are $3 \mathrm{D}$ position and $3 \mathrm{D}$ velocity information of the masers, conservative priors for the solar motion, the average source peculiar motion, and the Galactic scale and rotation. Convergence on the best Galactic parameters to match the spatialkinematic model was made using a Bayesian fitting approach, where the velocities were used as known data to be fitted, and the sky positions and distances were used as coordinates. The posterior probability density function (PDF) of the Galactic parameters were estimated with Markov chain Monte Carlo (MCMC) trials that were accepted or rejected by a Metropolis-Hastings algorithm (see Reid et al. 2009, 2014, for a detailed explanation). Finally, the procedure returns the best Galactic parameter values that match the simulated data to the spatial-kinematic model. The fitting procedure was improved compared to that used in Reid et al. (2009, 2014): first, the fitting procedure now corrects for bias when inverting parallax to estimate distance, which becomes significant when fractional parallax uncertainties exceet $\approx 15 \%$ (note this is not a trivial inference problem, see e.g. Bailer-Jones 2015); second, the fitting procedure was improved by adding a term to the motion uncertainties, which comes from parallax uncertainty. After these two modifications, the fitting procedure yielded unbiased Galactic parameter values, even when weak and/or very distant masers with large fractional parallax uncertainties were simulated.

\section{Results}

A comparison of the systematic $6.7 \mathrm{GHz}$ methanol maser observational surveys and the simulated model peak flux density is shown in Sect. 3.1. In Sect. 3.2, different sample selections are used to compare the Galactic parameters obtained with respect to the initial values used (Table 2). Finally, in Sect. 3.3, the Pearson correlation coefficients are calculated to quantify correlations among the Galactic parameters.

\subsection{Luminosity function for $6.7 \mathrm{GHz}$ methanol masers}

We compared the flux density distribution functions of the MMB survey and the Arecibo survey with the current model to fit two parameters: the total number of sources $(N)$ and the slope of 
Table 3. Limits in sensitivity and source location, numbers of masers detected and the slope of the flux density functions $(\beta)$ for the $6.7 \mathrm{GHz}$ methanol masers surveys: MMB and Arecibo.

\begin{tabular}{ccccc}
\hline \hline & \multicolumn{2}{c}{ MMB } & \multicolumn{2}{c}{ Arecibo } \\
& Observation & Simulation & Observation & Simulation \\
\hline Sensitivity $(3 \sigma)$ & \multicolumn{2}{c}{$\leq 0.71 \mathrm{Jy}$} & \multicolumn{2}{c}{$\leq 0.27 \mathrm{Jy}$} \\
\hline Sky & \multicolumn{2}{c}{$-174^{\circ} \leq l \leq 60^{\circ}$} & \multicolumn{2}{c}{$35.2^{\circ} \leq l \leq 53.7^{\circ}$} \\
coverage & $-2^{\circ} \leq b \leq 2^{\circ}$ & $-0.4^{\circ} \leq b \leq 0.4^{\circ}$ \\
\hline Sources & 908 & $800 \pm 20$ & 76 & $95 \pm 10$ \\
\hline$\beta$ & $-0.60 \pm 0.1$ & $-0.44 \pm 0.1$ & $-0.36 \pm 0.1$ & $-0.38 \pm 0.1$ \\
\hline
\end{tabular}

Notes. Limits of both surveys, numbers of masers $(N)$ and slope of the luminosity function $(\alpha)$ fitted in Sect. 3.1, were applied to our Galactic model; the results are displayed in the columns labeled "Simulation". The simulated errors correspond to the standard deviation after running 100 simulated galaxies.
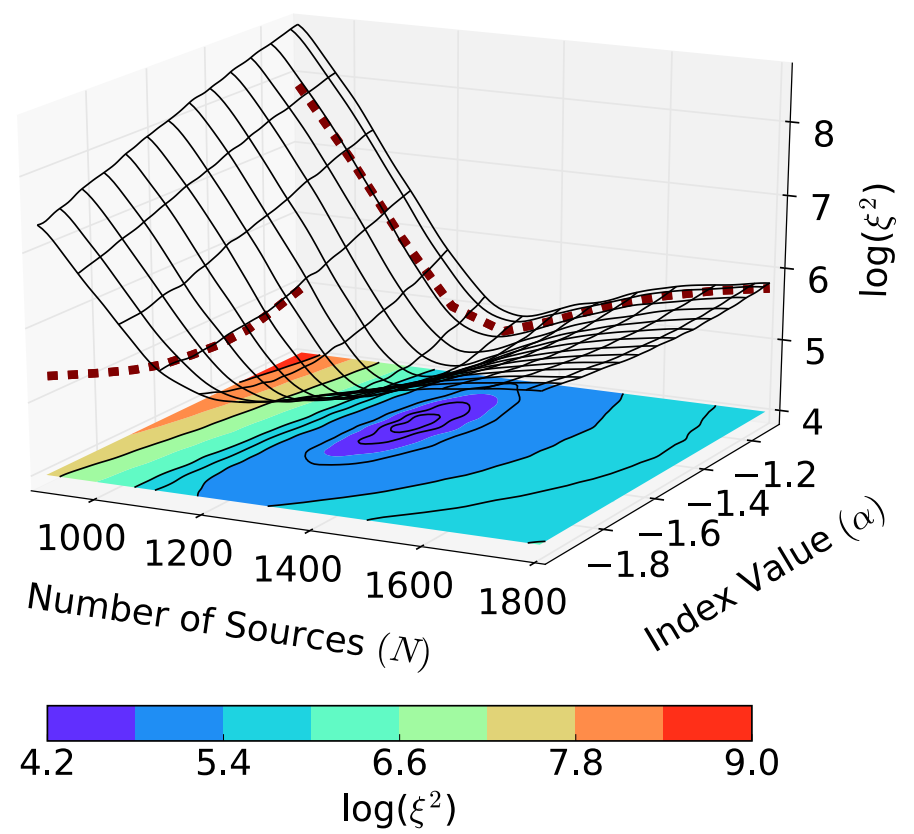

Fig. 5. Grid of initial parameters displaying the $\xi^{2}$ calculation for each $N, \alpha$ pair. The dark blue region represents the best values of $N$ and $\alpha$ that most closely match the MMB results. The projected gray dashed lines show the profiles of the surface close to the minimum values of $\xi^{2}$.

the peak luminosity function $(\alpha)$. The MMB survey is the most sensitive unbiased survey yet undertaken for $6.7 \mathrm{GHz}$ methanol masers. The Parkes Observatory was upgraded with a sevenbeam receiver to carry out a full systematic survey of the Galactic Plane (Green et al. 2012, and the references within). The Arecibo Survey was a deep a $6.7 \mathrm{GHz}$ methanol maser survey over a limited portion of the Galactic plane (Pandian et al. 2007).

Table 3 summarizes the survey limits in sensitivity and sky coverage for the MMB and Arecibo surveys. The last two rows list the number of sources detected and the slope of the flux density function $(\beta)$ for each survey. By using these data, we were able to make a direct comparison between the simulated and observed flux density functions for each survey (green and blue histograms in Fig. 6). For our comparison, we excluded MMB sources that reside inside a Galactocentric radius of $3 \mathrm{kpc}$ as this region is not part of the model.

In order to fit $N$ and $\alpha$ to the results of the surveys, a grid of initial parameters (Fig. 5) was sampled using similar ranges to those proposed by Pestalozzi et al. (2007) for $N=$ [900, 1800]

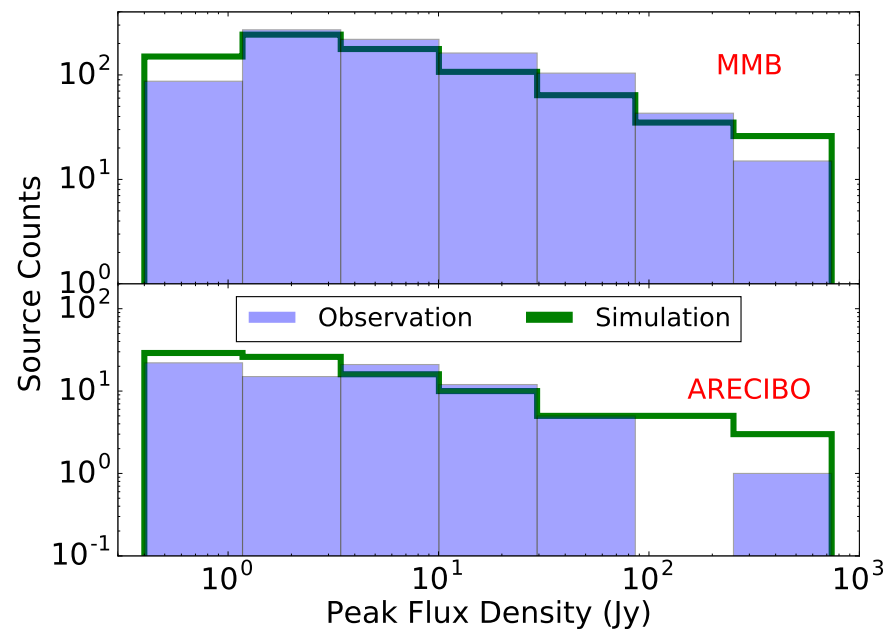

Fig. 6. In blue: flux density function obtained for the MMB (top) and the Arecibo survey (bottom). In green: simulated flux density function obtained in the model (using $N=1300$ and $\alpha=-1.43$ ) after the MMB and Arecibo limits were applied (Table 3 ).

and van der Walt (2005) for $\alpha=[-1.1,2.0]$. The grid was constructed such that each point represents a pair of initial parameters $(N, \alpha)$ and for each pair, a set of simulated galaxies was generated following the initial conditions described in Sect. 2. Next, the surveys limits (Table 3) were applied, and we compared the flux density function obtained for each $N, \alpha$ pair with the flux density function of the MMB survey (blue histogram in the top of Fig. 6). Through a minimization procedure, we found values of $N$ and $\alpha$ that best match the MMB results. This procedure was implemented only for the MMB data since it represents a larger and more complete sample than the Arecibo survey. The minimization procedure compares the MMB observed (blue) and the simulated (green) flux density functions (see Fig. 6) and minimizes a quantity called $\xi^{2}$, where

$\xi^{2}=\sum_{\text {bins }} \frac{\left(y-y_{\mathrm{obs}}\right)^{2}}{y_{\mathrm{obs}}}$,

and $y$ represents the number of sources per luminosity bin. Given that our Galactic model generates galaxies based on a stochastic method, the position, velocity and luminosity values for each maser vary each time the model is executed (even using the same pair of $N$ and $\alpha$ ). By generating sets of ten independent galaxy simulations per $N, \alpha$ pair, we found that the fluctuations in the simulations were smaller than the uncertainties in the binned data, and hence this procedure was applied.

Figure 5 shows the values obtained for $\xi^{2}$ per $N, \alpha$ pair as a $3 \mathrm{D}$ surface. The dark blue region in the projected contour plot represents the best set of parameters that mimic the MMB survey results. We found that the surface near the minimum can be approximated by a Gaussian in two dimensions (see projections in Fig. 5). Using the maximum likelihood estimation, which is well defined for multivariate Gaussian distributions, we estimated the mean and its respective uncertainty. The best parameters were found to be $N=1300 \pm 60$ sources and $\alpha=-1.43 \pm 0.18$. Finally, Fig. 6 shows the flux density function for the MMB (top), and Arecibo survey (bottom) in blue, and their respective simulated flux density function are shown in green for the best parameters of $N$ and $\alpha$ found. Additionally, the number of sources detected and the slope of the flux density function $(\beta)$ for the simulated surveys are listed in Table 3. 


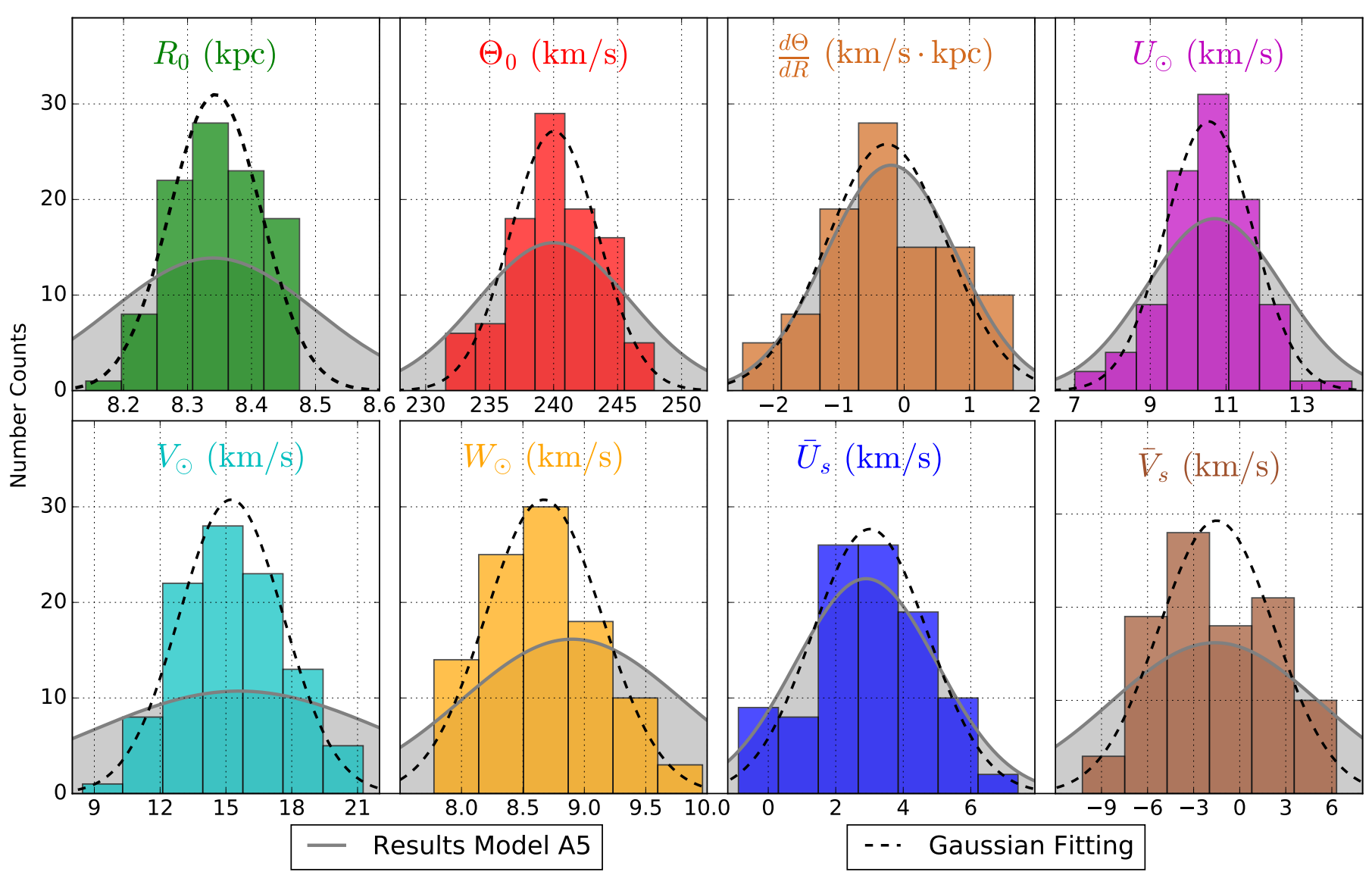

Fig. 7. Galactic parameters distributions found for 100 simulated galaxies mimicking the BeSSeL data sample selection (Sect. 3.2). The values listed in Table 4 correspond to the fitting made to the histograms and shown as black dashed lines. Bayesian fitting results for the A5 model reported in Reid et al. (2014) are shown as gray regions.

\subsection{Galactic parameters and selection of sample}

The model can reproduce the methanol maser distribution for the entire Galaxy including observational errors. In order to evaluate the possible biases introduced by the observed BeSSeL sample (equivalent to the 103 brightest sources in the declination region, $-30^{\circ} \leq \delta \leq 70^{\circ}$, which is equivalent to $-2^{\circ} \leq l \leq 242^{\circ}$ ), 100 galaxies were simulated to mimic the BeSSeL sample. Then, they were fitted to test whether the adopted Galactic parameters were returned. Figure 7 shows the distribution obtained on each Galactic parameter for the simulated BeSSeL sample compared with the values reported in Reid et al. (2014). The histograms were fitted to Gaussian distributions, and the results are shown in Table 4. Clearly, in all cases the distributions of fitted values are centered on the adopted value, and in most cases the widths of the distributions are smaller than those reported in Reid et al. (2014).

In addition to the 100 simulated galaxies that mimic the BeSSeL sample, we also simulated the first BeSSeL data sample, where only 16 HMSFRs over the northern hemisphere were used to estimate the same Galactic parameters but not the solar motion (Reid et al. 2009). Moreover, we also started adding sources to form two additional sets of simulated data. Set A was made to study the impact of future viable observations with the VLBA, EVN, and VERA to obtain up to 500 sources in the northern hemisphere. Again, we selected the brightest sources first to fall in the same declination range that BeSSeL is targeting for this. We generated samples from 16 up to 500 sources, which were drawn from the total number sources $(N=1300)$ that may lie
Table 4. Galactic parameter results for 100 simulated galaxies mimicking the BeSSeL data sample.

\begin{tabular}{ccc}
\hline \hline $\begin{array}{c}\text { Galactic } \\
\text { parameter }\end{array}$ & $\begin{array}{c}\text { Simulated } \\
\text { BeSSeL sample }\end{array}$ & $\begin{array}{c}\text { A5 } \\
\text { model }\end{array}$ \\
\hline$R_{0}\left(\mathrm{kpc}^{-1}\right)$ & $8.34 \pm 0.07$ & $8.34 \pm 0.16$ \\
$\Theta_{0}\left(\mathrm{~km} \mathrm{~s}^{-1}\right)$ & $240.0 \pm 3.4$ & $240.0 \pm 8.0$ \\
$\mathrm{~d} \Theta / \mathrm{d} R\left(\mathrm{~km} \mathrm{~s}^{-1} \mathrm{kpc}^{-1}\right)$ & $-0.3 \pm 0.9$ & $-0.2 \pm 0.4$ \\
$U_{\odot}\left(\mathrm{km} \mathrm{s}^{-1}\right)$ & $10.5 \pm 1.2$ & $10.7 \pm 1.8$ \\
$V_{\odot}\left(\mathrm{km} \mathrm{s}^{-1}\right)$ & $15.2 \pm 2.4$ & $15.6 \pm 6.8$ \\
$W_{\odot}\left(\mathrm{km} \mathrm{s}^{-1}\right)$ & $8.7 \pm 0.5$ & $8.9 \pm 2.1$ \\
$U_{\mathrm{s}}\left(\mathrm{km} \mathrm{s}^{-1}\right)$ & $3.0 \pm 1.7$ & $2.9 \pm 2.1$ \\
$V_{\mathrm{s}}\left(\mathrm{km} \mathrm{s}^{-1}\right)$ & $-1.5 \pm 3.8$ & $-1.5 \pm 6.8$ \\
\hline
\end{tabular}

Notes. Additionally, the Bayesian fitting results for the A5 model reported in Reid et al. (2014), which were also the initial values adopted in the model (Table 2), are shown for comparison.

in the declination range proposed. Set B represents the conditions for a more complete effort when VLBI arrays in the southern hemisphere can contribute to the astrometric sample. As was done in set A, we selected the brightest sources but now without declination limitation, generating samples from 16 up to the complete sample $(N=1300)$. Each additional sample in both sets was simulated for 100 galaxies. We note that in all cases the errors continued to be based on the VLBA observations characteristics. 
A\&A 604, A72 (2017)

Table 5. Pearson product-moment correlation coefficients calculated for 100 galaxies simulated to mimic the BeSSeL data sample selection.

\begin{tabular}{|c|c|c|c|c|c|c|c|c|}
\hline & $R_{0}$ & $\Theta_{0}$ & $\mathrm{~d} \Theta / \mathrm{d} R$ & $U_{\odot}$ & $V_{\odot}$ & $W_{\odot}$ & $\bar{U}_{\mathrm{s}}$ & $\bar{V}_{\mathrm{s}}$ \\
\hline$R_{0}$ & $1.00(1.00)$ & & & & & & & \\
\hline$\Theta_{0}$ & $0.48(0.47)$ & $1.00(1.00)$ & & & & & & \\
\hline $\mathrm{d} \Theta / \mathrm{d} R$ & $0.27(0.10)$ & $0.21(0.14)$ & $1.00(1.00)$ & & & & & \\
\hline$U_{\odot}$ & $0.32(0.45)$ & $0.01(0.24)$ & $0.08(-0.12)$ & $1.00(1.00)$ & & & & \\
\hline$V_{\odot}$ & $0.02(0.02)$ & $-0.73(-0.80)$ & $0.05(-0.01)$ & $0.05(-0.01)$ & $1.00(1.00)$ & & & \\
\hline$W_{\odot}$ & $-0.01(0.00)$ & $0.02(-0.01)$ & $-0.01(0.03)$ & $-0.06(-0.02)$ & $0.01(0.01)$ & $1.00(1.00)$ & & \\
\hline $\bar{U}_{\mathrm{s}}$ & $0.51(0.52)$ & $-0.09(0.17)$ & $0.01(-0.09)$ & $0.51(0.84)$ & $0.05(-0.01)$ & $-0.04(0.00)$ & $1.00(1.00)$ & \\
\hline $\bar{V}_{\mathrm{s}}$ & $0.09(0.00)$ & $0.62(0.81)$ & $-0.47(0.02)$ & $-0.16(-0.03)$ & $-0.68(-0.99)$ & $0.01(-0.01)$ & $0.13(0.03)$ & $1.00(1.00)$ \\
\hline
\end{tabular}

Notes. The respective Pearson coefficient reported in Reid et al. (2014) for the observed sample are listed in parentheses.

Figure 8 shows how the Galactic parameter values change as more sources are added to the sample selection for sets A and B. The dashed lines represent the initial values adopted in the model, and the error bars represent the standard deviation found for each parameter. The first and current BeSSeL results are also shown as stars and labeled following the same convention used in Reid et al. (2009, 2014), i.e., Fit 3 and Model A5 respectively.

Our objective was to investigate the accuracy with which the Galactic parameters can be recovered in the presence of measurement errors. It was therefore important that we verify the robustness of the fitting algorithm and its dependence on the choice of initial parameters. To make sure the fitting procedure recovers the Galactic parameters in an unbiased way over a large range, we ran the algorithm over a number of values in the multidimensional parameter space that defines our Galactic models. We varied the most relevant parameters over a broad range $( \pm \Delta$ and $\pm 3 \Delta$ for the obtained simulated BeSSeL values related in Table 4) and calculated a normalized difference between the input parameters and the returned fits. We found that indeed the fitting procedure can properly recover the starting values.

\subsection{Parameter correlations}

Using the Galactic parameter values obtained for 100 simulated galaxies mimicking the BeSSeL data sample selection, we calculated the Pearson product-moment correlation coefficients between all the parameters from the output distributions. The coefficients found are shown in Table 5; for comparison, the Pearson coefficient estimates reported in Reid et al. (2014) from the fitting procedure are also listed. Pearson coefficients in Reid et al. (2014) were calculated by MCMC trials, but in our case we have a large number of samples, which provides an independent way to estimate the correlations. Our findings seems to be consistent with the coefficients published in Reid et al. (2014).

We also estimated the Pearson coefficients variation as more sources are added to the sample selection. In order to see whether the dependence between various parameters can be reduced, we focused on the more correlated parameters reported in Reid et al. (2014), i.e., $r_{\left(R_{0}, \Theta_{0}\right)}, r_{\left(\Theta_{0}, V_{\odot}\right)}, r_{\left(\Theta_{0}, \bar{V}_{\mathrm{s}}\right)}, r_{\left(U_{\odot}, \bar{U}_{\mathrm{s}}\right)}$ and $r_{\left(V_{\odot}-\bar{V}_{\mathrm{s}}\right)}$. Figure 9 shows the Pearson coefficient evolution among these parameters in sets A and B. Moreover, the Pearson coefficients calculated for the complete sample and those published by Reid et al. (2014) are shown for comparison.

\section{Discussion}

\subsection{Luminosity function of $6.7 \mathrm{GHz}$ methanol masers}

We found that $N=1300 \pm 60$ and $\alpha=-1.43 \pm 0.18$ are the initial parameters that best match the MMB results. Using these values, the number of sources detected and the slope of the flux density function $(\beta)$ are slightly underestimated with respect to the observational survey results (see Table 3). This difference could be related to the contamination from inner Galaxy sources included in the MMB, which were not included in the simulation. This can account for approximately 100 sources in the $N$ estimate, producing a value of $N=$ $1300_{-160}^{+60}$. This estimate seems to be consistent with the initial calculation made by Green \& McClure-Griffiths (2011) of $N=1250$ and also with the minimum value settled by Pandian (2007) of $N=1125$. Moreover, Green \& McClure-Griffiths (2011) reported $\alpha=-1.44 \pm 0.4$ using kinematic distance resolution data from the International Galactic Plane Survey, which is very close to our calculation and also gives support to our estimate of $N$ since in our method the two quantities were fitted simultaneously.

There is no physical argument that predicts the luminosity function to be a single power law distribution. However, for the scope of this paper, we are only interested in deriving an empirical relation for the peak luminosity function for a population of 6.7 $\mathrm{GHz}$ methanol masers with the proper characteristics. Additionally, a single power law peak luminosity function appears to be consistent with the results obtained for different systematic surveys (including the Arecibo survey, see Fig. 6) and, for bright sources, it has been previously suggested by several authors (e.g. Pandian et al. 2007; Green \& McClure-Griffiths 2011).

\subsection{Galactic parameters analysis}

The different samples described in Sect. 3.2 were created to test how accurately the BeSSeL methodology can determine the Galactic parameters. When the sample testing was initially made using the same fitting procedure employed in Reid et al. (2009, 2014), the resulting parameters start deviating from the initial parameters when more sources were added. When sources with large fractional errors in parallax are numerous, we found that this biases the determination towards larger distances, resulting in parameters that map to a bigger Galaxy. This observational effect (see e.g. Bailer-Jones 2015) was corrected by allowing the fitting procedure to de-bias distance estimations based on measured parallax. We note that the improvements to the fitting code do not alter the results in Reid et al. (2014), which was based on the brighter sources.

Figures 7 and 8 summarize the Galactic parameters obtained compared with the initial values adopted (see Table 2), using the current and possible future samples. The results in Table 4 and Fig. 7 obtained for 100 simulated galaxies using the BeSSeL data sample selection show that the Galactic parameter values can be determined very robustly. Figure 8 shows that the Galactic parameter results for the simulated samples of 100 sources (current BeSSeL data) in sets A and B are already very close to 
L. H. Quiroga-Nuñez et al.: Simulated maser distribution to constrain Milky Way parameters
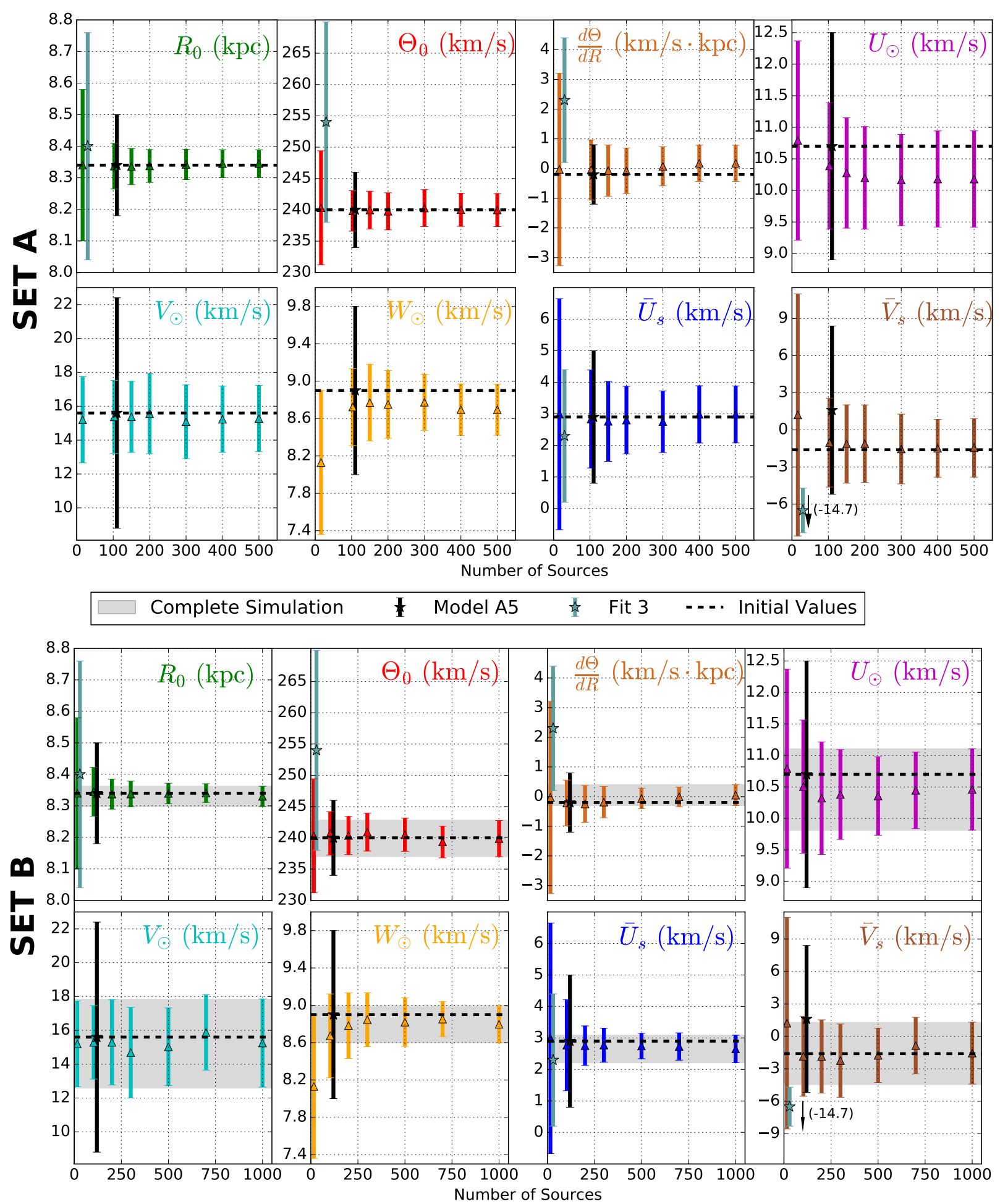

Fig. 8. Galactic parameter values obtained for samples in sets A and B. In each sample, sources are added in the northern hemisphere simulating the future BeSSeL results (set A) and without location limit simulating samples when southern arrays can contribute with data (set B). First and current BeSSeL results published in Reid et al. $(2009,2014)$, respectively labeled "Fit 3" and "Model A5", are shown as stars for comparison. The initial values adopted in the model are represented as dashed lines. Gray regions correspond to values and uncertainties obtained for the complete sample $(N=1300)$.

the initial parameters, and as more sources are added the uncertainties become smaller.

\subsubsection{Fundamental Galactic parameters: $R_{0}, \Theta_{0}$, and $\mathrm{d} \Theta / \mathrm{d} R$}

The differences in $R_{0}$ and $\Theta_{0}$ found when using 100 simulated galaxies mimicking the BeSSeL sample selection (Table 4 and
Fig. 7) are less than $0.2 \%$, demonstrating that indeed we can recover these parameters from the adopted model, even with samples that only contain northern hemisphere sources. Furthermore, the errors reported by Reid et al. (2014) for these parameters (i.e. $0.16 \mathrm{kpc}$ for $R_{0}$ and $8 \mathrm{~km} \mathrm{~s}^{-1}$ for $\Theta_{0}$ ), which are represented in Fig. 7 as gray regions, are double compared to our findings. Consequently, we conclude that the errors assigned 

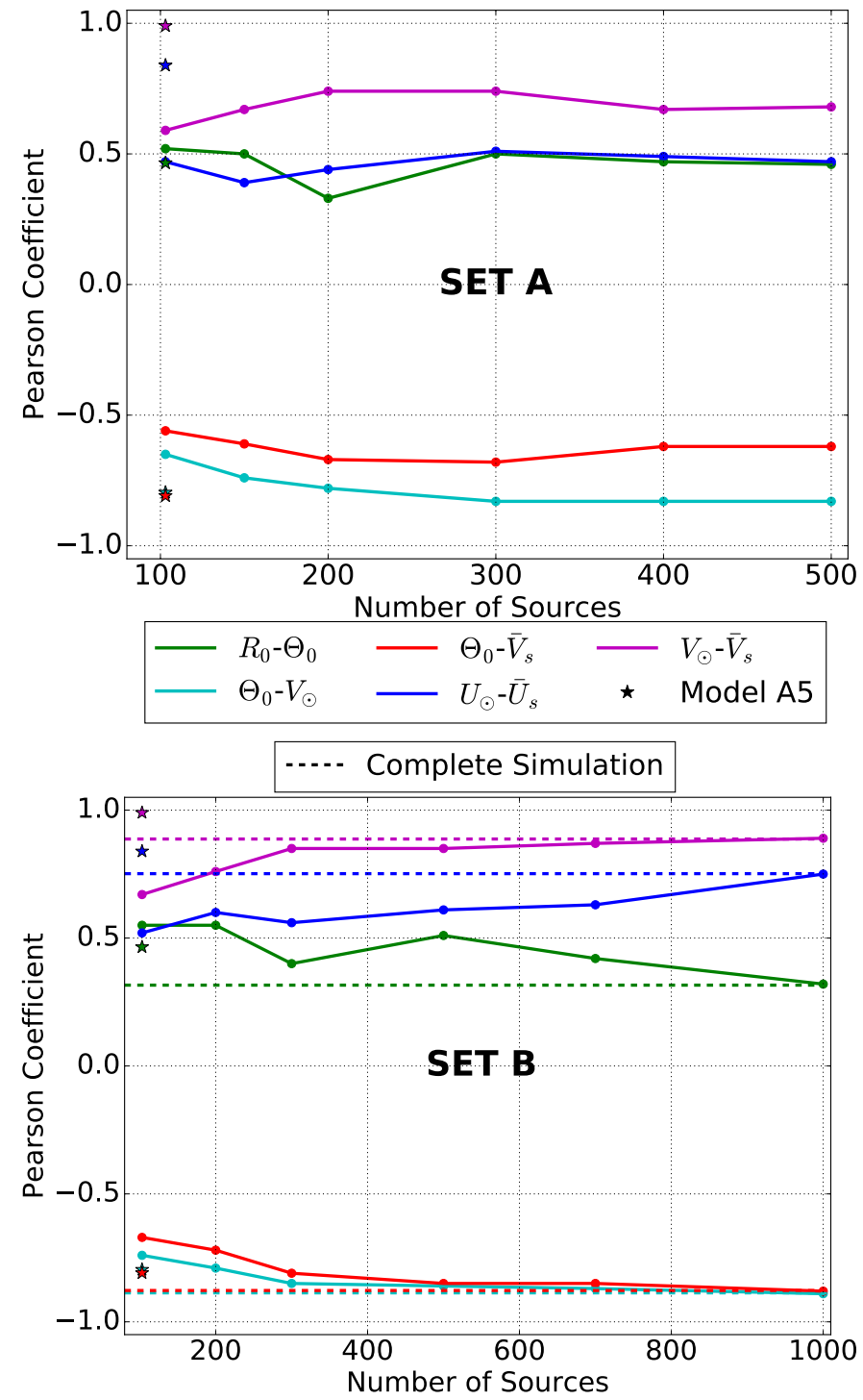

Fig. 9. Pearson product-moment correlation coefficients calculated for initially highly correlated values (see Table 5), when more sources are added in sets A (top panel) and B (bottom panel). Pearson coefficients reported by Reid et al. (2014) are shown as stars and those for the complete sample $(N=1300)$ are represented by dashed lines.

by Reid et al. (2014) to $R_{0}$ and $\Theta_{0}$ are conservative, and there do not appear to be any bias, given the available maser samples so far.

For the rotation curve, the situation is somewhat different. Although the values found for $\mathrm{d} \Theta / \mathrm{d} R$ are very close to the initial values adopted, the statistical spread is larger than expected. Reid et al. (2014) reported an error of $0.4 \mathrm{~km} \mathrm{~s}^{-1} \mathrm{kpc}^{-1}$ in the rotation curve which is optimistic compared with our findings. From our simulations, we would constrain the rotation curve value as $-0.3 \pm 0.9 \mathrm{~km} \mathrm{~s}^{-1} \mathrm{kpc}^{-1}$ given the BeSSeL data sample selection. The larger error possibly indicates that our assumed velocity distributions are too wide.

When more sources are added to the sample selection (sets A and B), Fig. 8 shows an initial improvement in the accuracy of the fundamental Galactic parameters. For set A, the errors in $R_{0}, \Theta_{0}$, and $\mathrm{d} \Theta / \mathrm{d} R$ can improve up to $\pm 0.04 \mathrm{kpc}, \pm 2.7 \mathrm{~km} \mathrm{~s}^{-1}$, and $\pm 0.6 \mathrm{~km} \mathrm{~s}^{-1} \mathrm{kpc}^{-1}$, respectively, when more northern hemisphere sources are added. In contrast when southern hemisphere sources are also added (set B), the errors can decrease to $\pm 0.03 \mathrm{kpc}, \pm 2.5 \mathrm{~km} \mathrm{~s}^{-1}$, and $\pm 0.3 \mathrm{~km} \mathrm{~s}^{-1} \mathrm{kpc}^{-1}$, respectively. Further improvements would require much better astrometry for weak sources, requiring for example much more sensitive observations.

\subsubsection{Solar motion and average source peculiar motions}

Figure 7 shows the distribution obtained for solar velocity components $\left(U_{\odot}, V_{\odot}\right.$, and $\left.W_{\odot}\right)$. The uncertainties derived were around $2 \%$ for all solar velocity components with respect to the initial parameters. For $U_{\odot}$, the standard deviation found was $1.2 \mathrm{~km} \mathrm{~s}^{-1}$, which compares well to the results published by Reid et al. (2014) (i.e., $U_{\odot}= \pm 1.8 \mathrm{~km} \mathrm{~s}^{-1}$ ). In addition, the spread for $V_{\odot}$ and $W_{\odot}$ are narrower with values of $2.4 \mathrm{~km} \mathrm{~s}^{-1}$ and $0.5 \mathrm{~km} \mathrm{~s}^{-1}$, respectively. Compared to the BeSSeL results (i.e., $V_{\odot}= \pm 6.8 \mathrm{~km} \mathrm{~s}^{-1}$ and $W_{\odot}= \pm 2.1 \mathrm{~km} \mathrm{~s}^{-1}$ ), we can affirm that the solar motion results published by Reid et al. (2014) have conservative estimates.

For the radial and tangential average peculiar motions $\left(\bar{U}_{\mathrm{s}}\right.$ and $\bar{V}_{\mathrm{s}}$ ), Table 4 shows that indeed these peculiar velocities can be fitted with high accuracy using the simulations; however, the relative spreads are high compared with other parameters (see Fig. 7). Even so, compared to the BeSSeL results (i.e., $\bar{U}_{\mathrm{s}}=$ $\pm 2.1 \mathrm{~km} \mathrm{~s}^{-1}$ and $\bar{V}_{\mathrm{s}}= \pm 6.8 \mathrm{~km} \mathrm{~s}^{-1}$ ), the errors in the simulated sample selection are still lower (i.e., $\bar{U}_{\mathrm{s}}= \pm 1.7 \mathrm{~km} \mathrm{~s}^{-1}$ and $\bar{V}_{\mathrm{s}}=$ $\pm 3.8 \mathrm{~km} \mathrm{~s}^{-1}$ ).

The reason for higher dispersions for $\bar{U}_{\mathrm{s}}$ and $\bar{V}_{\mathrm{s}}$ could be related to the number of parameters that must fit independently. The parameters $\bar{U}_{\mathrm{s}}$ and $U_{\odot}$ have largely the same effect on the observations for nearby sources and therefore are directly correlated (see Table 5). For $\bar{V}_{\mathrm{s}}$, the correlation is high with two components $\left(V_{\odot}, \Theta_{0}\right)$, which affects the fitting and hence the estimated accuracy.

\subsubsection{Parameter correlations}

Pearson product-moment correlation coefficients are shown in Table 5. All the parameters are in agreement with those found by Reid et al. (2014), except for the Pearson coefficient between $\mathrm{d} \Theta / \mathrm{d} R$ and $\bar{V}_{\mathrm{s}}$ where a low correlation was found in the observed sample instead of the moderate correlation found in the simulated sample. However, we focus the discussion on the parameters that were reported to have considerable correlation in Reid et al. (2014), as it is interesting to see how it is possible to disentangle these fundamental parameters.

For the first BeSSeL summary paper (Reid et al. 2009), where only 16 HMSFRs were used, the estimated $R_{0}$ and $\Theta_{0}$ were strongly correlated $\left(r_{R_{0}, \Theta_{0}}=0.87\right)$. Later on, in Reid et al. (2014), using a larger sample of 103 HMSFRs spanning a greater Galactic distribution, the correlation was significantly lower $\left(r_{R_{0}, \Theta_{0}}=0.47\right)$. We calculated the same coefficient by mimicking both samples using 100 simulated galaxies, finding similar results for each sample, i.e, $r_{R_{0}, \Theta_{0}}=0.77$ and $r_{R_{0}, \Theta_{0}}=0.48$. These results show that, indeed, our simulation produces similar correlation coefficients to those found in the observations, even when the method used to calculate the Pearson coefficients are completely different (see Sect. 3.3). When more sources are added to the sample selection, Fig. 9 shows that the correlation between $R_{0}$ and $\Theta_{0}$ is reduced. Furthermore, the Pearson coefficient will have a moderate value $(0.3)$ when using the complete data sample, which demonstrates that the correlation between these parameters can be unraveled smoothly as more sources are added. 

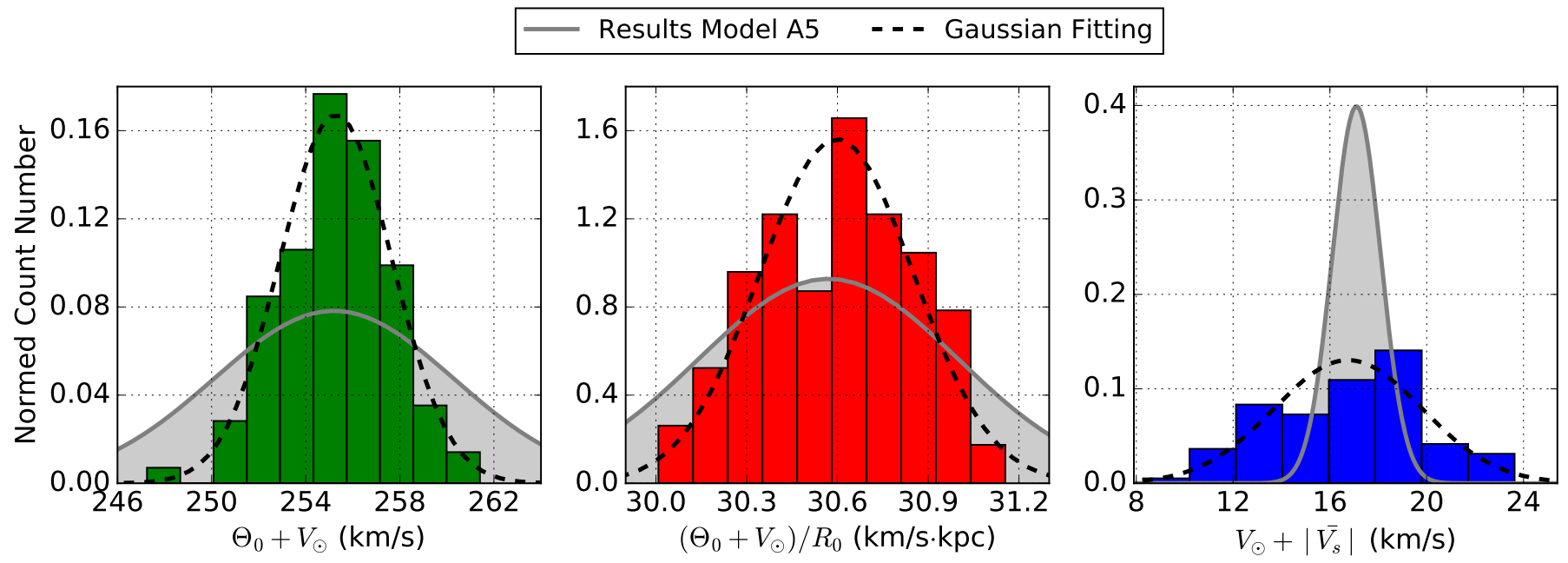

Fig. 10. Marginalized posterior probability density distributions for correlated circular velocity parameters from 100 simulated galaxies mimicking the BeSSeL sample. Left panel: circular orbital speed of the Sun. Middle panel: orbital angular solar speed. Right panel: difference between the circular solar motion and average source peculiar motions.

For the tangential velocity component, we have three different Galactic parameters giving similar effects: $\Theta_{0}, V_{\odot}$ and $\bar{V}_{\mathrm{s}}$. Figure 9 and Table 5 show that the Pearson coefficients among these parameters are always high, even when more sources are added. This implies that different types of data are needed in order to better disentangle these Galactic parameters. Finally, in the radial direction we have two Galactic parameters: $U_{\odot}$ and $\bar{U}_{\mathrm{s}}$. The correlation between these parameters is around 0.5 for a low number of sources, as it shown in Fig. 9 and listed in Table 5. When more sources are added, the correlation parameter seems to maintain the same value or slightly increase in both sets of samples (see Fig. 9). One could expect that the inclusion of southern hemisphere sources would help to disentangle some of the dependences; however, comparing the top and bottom plots in Fig. 9 we can see that using samples with a larger coverage of the Galaxy does not alter significantly the correlation values found for any of the Pearson coefficients discussed here.

As some of the parameter correlations persist, even when more sources are added to the sample selection, we estimated the marginalized PDFs for different combined parameters that were also reported in the latest BeSSeL survey paper. Figure 10 shows the PDFs for the circular orbital speed of the Sun, the orbital angular solar speed, and the difference between the circular solar and average source peculiar motions. Additionally, those PDFs reported by Reid et al. (2014) are shown as gray regions.

We found $255.3 \pm 2.4 \mathrm{~km} \mathrm{~s}^{-1}, 30.60 \pm 0.26 \mathrm{~km} \mathrm{~s}^{-1} \mathrm{kpc}^{-1}$, and $16.7 \pm 3.1 \mathrm{~km} \mathrm{~s}^{-1}$, respectively, for the correlated values of the parameters $\Theta_{0}+V_{\odot},\left(\Theta_{0}+V_{\odot}\right) / R_{0}$ and $V_{\odot}+\left|\bar{V}_{\mathrm{s}}\right|$. Reid et al. (2014) found more conservative values for $\Theta_{0}+V_{\odot}=255.2 \pm 5.1 \mathrm{~km} \mathrm{~s}^{-1}$ and $\left(\Theta_{0}+V_{\odot}\right) / R_{0}=30.57 \pm 0.43 \mathrm{~km} \mathrm{~s}^{-1} \mathrm{kpc}^{-1}$ than we did. Although the mean value found for $V_{\odot}+\left|\bar{V}_{\mathrm{s}}\right|$ is in agreement with the BeSSeL results (i.e., $17.1 \pm 1.0 \mathrm{~km} \mathrm{~s}^{-1}$ ), we estimate a wider error of $\pm 3.1 \mathrm{~km} \mathrm{~s}^{-1}$ based on our simulations.

\section{Conclusions}

We constructed simulations of $6.7 \mathrm{GHz}$ methanol maser distributions to test whether the Galactic parameter results obtained by the BeSSeL survey (Reid et al. 2014) are biased in any way and investigated the interdependencies between some parameter estimates. We used our model to constrain the peak flux density function for the masers and obtained similar results to those of systematic unbiased surveys (MMB and Arecibo). This comparison allowed us to estimate the integral number of sources $\left(N=1300_{-160}^{+60}\right)$ and the slope of the luminosity function $(\alpha=-1.43 \pm 0.18)$, which showed good agreement with Pandian (2007), Green \& McClure-Griffiths (2011), Urquhart et al. (2013).

Assuming that the observations are predominantly of $12 \mathrm{GHz}$ methanol masers found through $6.7 \mathrm{GHz}$ surveys, we simulated the current database of the BeSSeL survey hundreds of times. We found that the fundamental Galactic parameters $\left(R_{0}, \Theta_{0}\right.$, $\mathrm{d} \Theta / \mathrm{d} R)$, the solar velocity components $\left(U_{\odot}, V_{\odot}, W_{\odot}\right)$ and the average peculiar motion $\left(\bar{U}_{\mathrm{s}}, \bar{V}_{\mathrm{s}}\right)$ can be determined robustly. Furthermore, the results published by Reid et al. (2014) have a conservative error calculation given the current sample, except possibly for the rotation curve error estimate. Also, correlation coefficients for the various Galactic parameters in our simulations and those reported by Reid et al. (2014) are similar.

Additionally, the fitting procedures developed by Reid et al. $(2009,2014)$ for use with the BeSSeL data and improved in this study estimate Galactic parameters correctly even when weak and/or distant sources with large fractional parallax uncertainties are included in the samples.

For future BeSSeL observations, the simulations demonstrate that the Galactic parameter estimates can be improved and the error bars reduced significantly. Moreover, using southern hemisphere data, the Galactic parameter estimates improve notably compared with samples limited to the northern sky.

We find that the uncertainties in the values of certain combined velocity parameters that are highly correlated are similar to those published in Reid et al. (2014), except for the dispersion in $V_{\odot}+\left|\bar{V}_{\mathrm{s}}\right|$. However, when more sources are added to the sample, the correlations among most Galactic parameters are smoothly reduced; for the highly velocity parameters the correlation coefficients do not decrease significantly.

The framework proposed to test the results of the BeSSeL survey is useful for defining requirements for future astrometric campaigns that are similar or complementary to the BeSSeL data. Southern arrays - like the Australian Long Baseline Array (see e.g. Krishnan et al. 2015, 2017) and, in the future, the African VLBI Network and the Square Kilometre Array in 
Australia and South Africa - will supplement the lack of precise astrometric data in quadrants III and IV of the Milky Way plane, where only a few sources have been measured. Moreover, astrometric studies that include the inner Galactic region, such as the Bulge Asymmetries and Dynamic Evolution (BAaDE $\left.{ }^{2}\right)$ project, aim to resolve the dynamics of the bar by measuring proper motions and distances of $\mathrm{SiO}$ masers present in AGB stars (Sjouwerman et al. 2017). Out of the Galactic plane, Gaia will soon provide astrometric results for a large number of sources. All of these investigations will contribute to the determination of Galactic parameters with even better accuracy with new and improved astrometric data. Until then, Galactic simulations complement the current observations by demonstrating their robustness and potential.

Acknowledgements. We sincerely thank the anonymous referee for making valuable suggestions that have improved the paper. L.H.Q.-N. acknowledges the comments and suggestions regarding the model implementation made by $\mathrm{S}$. Solorzano-Rocha at ETH Zürich.

\section{References}

Bailer-Jones, C. A. L. 2015, PASP, 127, 994

Bobylev, V. V., \& Bajkova, A. T. 2016, Astron. Lett., 42, 182

Bovy, J., \& Rix, H.-W. 2013, ApJ, 779, 115

Breen, S. L., Ellingsen, S. P., Contreras, Y., et al. 2013, MNRAS, 435, 524

Brunthaler, A., Reid, M. J., Menten, K. M., et al. 2011, Astron. Nachr., 332, 461

Burns, R. A., Handa, T., Imai, H., et al. 2017, MNRAS, 467, 2367

Caswell, J. L., Fuller, G. A., Green, J. A., et al. 2010, MNRAS, 404, 1029
Caswell, J. L., Fuller, G. A., Green, J. A., et al. 2011, MNRAS, 417, 1964 Cheng, J. Y., Rockosi, C. M., Morrison, H. L., et al. 2012, ApJ, 752, 51 Dame, T. M., Hartmann, D., \& Thaddeus, P. 2001, ApJ, 547, 792 Efremov, Y. N. 2011, Astron. Rep., 55, 108

Gaia Collaboration (Brown, A. G. A., et al.) 2016, A\&A, 595, A2

Gilmore, G., \& Reid, N. 1983, MNRAS, 202, 1025

Goodman, A. A., Alves, J., Beaumont, C. N., et al. 2014, ApJ, 797, 53

Green, J. A., \& McClure-Griffiths, N. M. 2011, MNRAS, 417, 2500

Green, J. A., Caswell, J. L., Fuller, G. A., et al. 2009, MNRAS, 392, 783

Green, J. A., Caswell, J. L., Fuller, G. A., et al. 2010, MNRAS, 409, 913

Green, J. A., Caswell, J. L., McClure-Griffiths, N. M., et al. 2011, ApJ, 733, 27

Green, J. A., Caswell, J. L., Fuller, G. A., et al. 2012, MNRAS, 420, 3108

Krishnan, V., Ellingsen, S. P., Reid, M. J., et al. 2015, ApJ, 805, 129

Krishnan, V., Ellingsen, S. P., Reid, M. J., et al. 2017, MNRAS, 465, 1095

Pandian, J. D. 2007, Ph.D. Thesis, Cornell University, USA

Pandian, J. D., Goldsmith, P. F., \& Deshpande, A. A. 2007, ApJ, 656, 255

Perryman, M. A. C., Lindegren, L., Kovalevsky, J., et al. 1997, A\&A, 323, L49

Perryman, M. A. C., de Boer, K. S., Gilmore, G., et al. 2001, A\&A, 369, 339

Pestalozzi, M. R., Chrysostomou, A., Collett, J. L., et al. 2007, A\&A, 463, 1009

Reid, M. J., \& Honma, M. 2014, ARA\&A, 52, 339

Reid, M. J., Menten, K. M., Zheng, X. W., et al. 2009, ApJ, 700, 137

Reid, M. J., Menten, K. M., Brunthaler, A., et al. 2014, ApJ, 783, 130

Rix, H.-W., \& Bovy, J. 2013, A\&ARv, 21, 61

Sanna, A., Reid, M. J., Menten, K. M., et al. 2014, ApJ, 781, 108

Sjouwerman, L. O., Pihlström, Y. M., Rich, R. M., Morris, M. R., \& Claussen, M. J. 2017, in The Multi-Messenger Astrophysics of the Galactic Centre, eds. R. M. Crocker, S. N. Longmore, \& G. V. Bicknell, IAU Symp., 322, 103

Surcis, G., Vlemmings, W. H. T., van Langevelde, H. J., Hutawarakorn Kramer, B., \& Quiroga-Nuñez, L. H. 2013, A\&A, 556, A73

Urquhart, J. S., Moore, T. J. T., Schuller, F., et al. 2013, MNRAS, 431, 1752

van der Walt, J. 2005, MNRAS, 360, 153

Wainscoat, R. J., Cohen, M., Volk, K., Walker, H. J., \& Schwartz, D. E. 1992, ApJS, 83, 111

Yusof, N., Hirschi, R., Meynet, G., et al. 2013, MNRAS, 433, 1114

2 http://www.phys.unm. edu/ baade/index.html 